\title{
Improved data for solar flare X-ray spectral analysis
}

\author{
K. J. H. Phillips ${ }^{1}$, J. A. Rainnie ${ }^{2}$, L. K. Harra ${ }^{3}$, J. Dubau ${ }^{4}$, F. P. Keenan ${ }^{4}$, and N. J. Peacock ${ }^{6}$ \\ 1 National Research Council Senior Research Associate, NASA Goddard Space Flight Center, Greenbelt, MD 20771, USA \\ 2 Space Science and Technology Dept., Rutherford Appleton Laboratory, Chilton, Didcot, Oxon OX11 0QX, UK \\ ${ }^{3}$ Mullard Space Science Laboratory, University College London, Holmbury St Mary, Dorking, Surrey RH5 6NT, UK \\ 4 Observatoire de Paris-Meudon, Place Jules Janssen, 92195 Meudon, France \\ 5 Dept. of Physics, The Queen's University, Belfast BT7 1NN, N. Ireland, UK \\ ${ }^{6}$ EURATOM/UKAEA Fusion Association, Culham Science Centre, Abingdon, Oxon OX14 3DB, UK
}

Received 12 September 2003 / Accepted 10 November 2003

\begin{abstract}
The inclusion of collisional rates for He-like Fe and $\mathrm{Ca}$ ions is discussed with reference to the analysis of solar flare Fe XXV and Ca XIX line emission, particularly from the Yohkoh Bragg Crystal Spectrometer (BCS). The new data are a slight improvement on calculations presently used in the BCS analysis software in that the discrepancy in the Fe XXV $y$ and $z$ line intensities (observed larger than predicted) is reduced. Values of electron temperature from satellite-to-resonance line ratios are slightly reduced (by up to $1 \mathrm{MK}$ ) for a given observed ratio. The new atomic data will be incorporated in the Yohkoh BCS databases. The data should also be of interest for the analysis of high-resolution, non-solar spectra expected from the Constellation- $X$ and Astro- $E$ space missions. A comparison is made of a tokamak S XV spectrum with a synthetic spectrum using atomic data in the existing software and the agreement is found to be good, so validating these data for particularly high- $n$ satellite wavelengths close to the S XV resonance line. An error in a data file used for analyzing BCS Fe XXVI spectra is corrected, so permitting analysis of these spectra.
\end{abstract}

Key words. Sun: flares - Sun: X-rays, gamma rays - X-rays: spectra

\section{Introduction}

For over ten years, from the time of its launch on 1991 August 30 till the loss of communications on 2001 December 14, the Yohkoh spacecraft observed solar activity, recording images in soft and hard X-rays with the Soft Xray Telescope and Hard X-ray Telescope, and recording Xray spectra with the Bragg Crystal Spectrometer (BCS) and Wide Band Spectrometer (see Martens \& Cauffman 2002 for a summary of Yohkoh discoveries). Over its lifetime, the BCS recorded X-ray spectra from many thousands of flares. Analysis of flare and active region spectra from the BCS has led to the determination of parameters that characterize the emitting plasma. This was achieved through the observation of resonance lines of He-like Fe, Ca, and $\mathrm{S}$ (Fe XXV, Ca XIX, S XV), $\mathrm{H}$-like $\mathrm{Fe}$ (Fe XXVI), and nearby dielectronic satellite lines. The intensity ratios of satellites to resonance lines give electron temperatures $T_{\mathrm{e}}$ and emission measures $E M=\int_{V} N_{\mathrm{e}}^{2} \mathrm{~d} V$ $\left(N_{\mathrm{e}}=\right.$ electron density, $V=$ source volume $)$, while line

Send offprint requests to: K. J. H. Phillips, e-mail: phillips@stars.gsfc.nasa.gov profiles and displacements from rest positions give details about directed and random plasma motions.

Analysis of BCS data requires databases containing instrument parameters and atomic data referring to the ions and spectral lines, and computer routines calculating theoretical spectra. The routines, written in Interactive Data Language (IDL) and incorporated into the SolarSoft package, read the input data, build spectral files for each of the four BCS channels, and iteratively fit the observed spectra to theoretical spectra. The output consists of values of $T_{\mathrm{e}}, E M$, nonthermal velocities (from line widths) and any directed velocities (in the case of twocomponent spectra), and the value of the reduced $\chi^{2}$. Much of the atomic data was assembled in the early 1980's for analysis of spectra from the Bent Crystal Spectrometer on Solar Maximum Mission (SMM), operational from 1980 to 1989. Since then improved data for particularly the collisional excitation rates have become available. In this paper, we show the effects of these data on the analysis of solar flare Fe XXV and Ca XIX spectra (Sect. 3) and with observations from an electron beam ion trap (Sect. 4). We also discuss a synthetic S XV spectrum based on the BCS software which is compared with a high-resolution tokamak spectrum (Sect. 5). Finally, we resolve a long-standing error in the software that has prevented routine analysis of Fe XXVI line emission in flares (Sect. 6). 


\section{The Yohkoh Bragg Crystal Spectrometer and SMM Bent Crystal Spectrometer}

The Yohkoh BCS (Culhane et al. 1991) consists of a pair of spectrometers, each pair having a single position-sensitive detector viewing spectra which are Bragg-diffracted by two germanium crystals. With the slightly bent (but non-focussing) crystals, the Bragg diffraction condition, $n \lambda=2 d \sin \theta(\theta=$ Bragg angle, $n=$ diffraction order, $d=$ crystal lattice spacing) varies over the face of each crystal. The BCS has no collimator, this having the advantage of improved sensitivity over the $S M M$ Bent Crystal Spectrometer and other similar spectrometers, but the disadvantage, with a whole-Sun field of view, of spectral confusion when more than one emitting region was present. This is a particular problem for the S XV channel which is sensitive to emission of small flares and non-flaring active regions. For an on-axis source, the wavelength ranges are 1.764$1.804 \AA$ (channel 1: Fe XXVI lines), 1.830-1.894 $\AA$ (channel 2: Fe XXV lines), 3.163-3.191 ^ (channel 3: Ca XIX lines), 5.006$5.114 \AA$ (channel 4: S XV lines).

Because of the spectrometers' arrangement, spectra could be formed simultaneously over integration times as small as $9 \mathrm{~s}$. The spectral resolution is determined by both the crystal rocking curves and noise in the various electronic systems, particularly the detectors. The resolution of the Fe XXV and Ca XIX channels appears to be worse than indicated from pre-launch measurements (Mariska \& Doschek 1997; Kepa et al. 1999): best estimates for the FWHM are $2 \mathrm{~mA}$ (instead of the stated $0.53 \mathrm{~m} \AA$ ) and $1 \mathrm{~m} \AA$ (instead of the stated $0.53 \mathrm{~m} \AA$ ). At very high count rates both detectors $A$ and $B$ saturated, i.e. an excessive number of photon counts leading to the detectors failing to count photons accurately. This meant that relatively few flares were observed with significant Fe XXVI line emission, since these lines are only emitted at very high temperatures, normally occurring at times of very high X-ray intensities which cause detector A to saturate (Pike et al. 1996). A background always present in channels 1 and 2 spectra is due to fluorescence of the germanium crystals by solar X-rays with energies greater than $11 \mathrm{keV}$. This background was electronically eliminated for channels 3 and 4 so the solar flare continuum can be observed in these channels.

The Bent Crystal Spectrometer on the SMM spacecraft (Acton et al. 1980), which functioned between 1980 and 1989, was of similar design to the Yohkoh BCS but the detectors worked on a different principle. There were eight channels (covering the Fe XXV and Fe XXVI lines and nearby satellites and the Ca XIX lines) instead of four, and the spectrometer viewed solar X-rays through a 6 arcmin FWHM collimator. The Fe XXV channel had a spectral resolution of $0.43 \mathrm{~m} \AA$. As this is better than the equivalent Yohkoh BCS channel, we shall compare theoretical Fe XXV spectra in this discussion with $S M M$ spectra.

\section{Solar flare Fe XXV and Ca XIX spectra}

The principal lines of Fe XXV and CaXIX are due to transitions $1 \mathrm{~s}^{2}{ }^{1} \mathrm{~S}_{0}-1 \mathrm{~s} 2 \mathrm{p}^{1} \mathrm{P}_{1}$ (resonance line, or line $w$ in the notation of Gabriel 1972), $1 \mathrm{~s}^{2}{ }^{1} \mathrm{~S}_{0}-1 \mathrm{~s} 2 \mathrm{p}^{3} \mathrm{P}_{2}(x), 1 \mathrm{~s}^{2}{ }^{1} \mathrm{~S}_{0}-$ $1 \mathrm{~s} 2 \mathrm{p}^{3} \mathrm{P}_{1}(y)$, and $1 \mathrm{~s}^{2}{ }^{1} \mathrm{~S}_{0}-1 \mathrm{~s} 2 \mathrm{~s}^{3} \mathrm{~S}_{1}(z)$. Dielectronic satellites with transitions $1 \mathrm{~s}^{2} n l-1 \mathrm{~s} 2 \mathrm{p} n l(n \geq 2, l=\mathrm{s}$, p etc.) occur on the long-wavelength side of $w$. Those in arrays $1 \mathrm{~s}^{2} n \mathrm{p}-1 \mathrm{~s} 2 \mathrm{p} n \mathrm{p}$ $(n \geq 2)$ are formed by dielectronic capture of a free electron by the He-like stage to form a doubly excited state which stabilizes by radiative de-excitation (dielectronic recombination). Satellite lines in the $1 s^{2} 2 s-1 s 2 s 2 p$ array are formed by both dielectronic recombination of the He-like stage and inner-shell excitation of the Li-like stage. Examples of intense dielectronic recombination satellites are $j\left(1 \mathrm{~s}^{2} 2 \mathrm{p}{ }^{2} \mathrm{P}_{3 / 2}-1 \mathrm{~s} 2 \mathrm{p}^{2}{ }^{2} \mathrm{D}_{5 / 2}\right)$ and $k\left(1 \mathrm{~s}^{2} 2 \mathrm{p}^{2} \mathrm{P}_{1 / 2}-1 \mathrm{~s} 2 \mathrm{p}^{2}{ }^{2} D_{3 / 2}\right)$; examples of inner-shell satellites are $q\left(1 \mathrm{~s}^{2} 2 \mathrm{~s}^{2} \mathrm{~S}_{1 / 2}-1 \mathrm{~s} 2 \mathrm{~s} 2 \mathrm{p}^{2} \mathrm{P}_{3 / 2}\right)$ and $r\left(1 \mathrm{~s}^{2} 2 \mathrm{~s}^{2} \mathrm{~S}_{1 / 2}-\right.$ $\left.1 \mathrm{~s} 2 \mathrm{~s} 2 \mathrm{p}^{2} \mathrm{P}_{1 / 2}\right)$. The wavelength range of the Yohkoh BCS channel 2 includes the Fe XXV $w-z$ lines and all the main Fe XXIV dielectronic satellites, but BCS channel 3 includes only the Ca XIX $w$ and $x$ lines (occasionally the $y$ line also, depending on the flare's position with respect to the BCS optical axis) and a line feature composed of the $d 13$ and $d 15 n=3$ satellites (notation of Bely-Dubau et al. 1979). The corresponding $S M M$ spectrometer observed Fe XXV $w-z$ lines and associated Fe XXIV satellites as well as satellites from lower stages (down to $\mathrm{Fe} \mathrm{XX)}$ and the Fe fluorescence $\mathrm{K} \alpha$ lines at 1.936, $1.940 \AA$. The SMM spectrometer also observed Ca XIX lines $w-z$ and associated Ca XVIII satellites.

The diagnostic importance of Fe XXV and Ca XIX spectra lies in the fact that the intensity ratio of a dielectronic recombination satellite to the resonance line, $I_{\mathrm{s}} / I_{\mathrm{w}}$, depends on $T_{\mathrm{e}}$ according to

$\frac{I_{\mathrm{s}}}{I_{\mathrm{w}}}=\frac{F(s) \exp \left[\left(E_{0}-E_{\mathrm{s}}\right) / k_{\mathrm{B}} T_{\mathrm{e}}\right]}{T_{\mathrm{e}}}$,

where $E_{0}$ is the $w$ line excitation energy, $E_{\mathrm{s}}$ is the excitation energy of the upper level of the satellite line with respect to the $1 \mathrm{~s}^{2}{ }^{1} \mathrm{~S}_{0}$ level of the He-like ion, $k_{\mathrm{B}}$ is Boltzmann's constant, and $F(s)$ is an intensity factor given by $F(s)=A_{\mathrm{r}} A_{\mathrm{a}} /\left(\Sigma A_{\mathrm{r}}+A_{\mathrm{a}}\right)$, where $A_{\mathrm{r}}$ is the radiative transition probability for the satellite transition, $\Sigma A_{\mathrm{r}}$ the sum of all radiative transitions from the upper level, and $A_{\mathrm{a}}$ is the auto-ionization probability from the satellite's upper level. (Since $E_{0}-E_{\mathrm{s}} \lesssim k_{\mathrm{B}} T_{\mathrm{e}}, I_{\mathrm{s}} / I_{\mathrm{w}}$ is approximately proportional to $T_{\mathrm{e}}^{-1}$.) Dielectronic recombination satellites are formed from the recombination of the He-like ion stage, and so $I_{\mathrm{s}} / I_{\mathrm{w}}$ does not depend on the ratio of ionization stages, only on $T_{\mathrm{e}}$ and atomic factors (Eq. (1)). In general $A_{\mathrm{r}}>A_{\mathrm{a}}, A_{\mathrm{r}} \propto Z^{4}$, and $A_{\mathrm{a}}$ only weakly depends on $Z$ ( $Z=$ atomic number); the result is that satellites such as $j$ and $k$ are much more intense relative to line $w$ for $\mathrm{Fe} \mathrm{XXV} \mathrm{than} \mathrm{for}$ Ca XIX and lower- $Z$ elements $\left(I_{\mathrm{s}} / I_{\mathrm{w}}\right.$ has a roughly $Z^{4}$ dependence, but there is a turnover for elements with $Z$ greater than $\mathrm{Fe})$. Fe XXV spectra therefore offer an important temperature diagnostic for solar flare and tokamak plasmas.

The upper levels of each of the He-like ion lines $1 \mathrm{~s} 2 \mathrm{p}^{1} \mathrm{P}_{1}$, $1 \mathrm{~s} 2 \mathrm{p}^{3} \mathrm{P}_{1,2}$, and $1 \mathrm{~s} 2 \mathrm{~s}^{3} \mathrm{~S}_{1}$, are excited principally by electron collisions. The excitation may occur direct from the ground state $1 \mathrm{~s}^{2}{ }^{1} \mathrm{~S}_{0}$ to the upper level or from the ground state to levels higher than the upper level, followed by cascades to the upper level. Excitation may also proceed by radiative recombination of the H-like ion, $\mathrm{H}(1 \mathrm{~s})+\mathrm{e}^{-} \rightarrow \mathrm{He}(1 \mathrm{~s} 2 \mathrm{l})+h v$, dielectronic 
recombination of the $\mathrm{H}$-like ion, and for line $z$ by inner-shell ionization of the Li-like stage, $\operatorname{Li}\left(1 \mathrm{~s}^{2} 2 \mathrm{~s}\right)+\mathrm{e}_{1}^{-} \rightarrow \mathrm{He}\left(1 \mathrm{~s} 2 \mathrm{~s}^{3} \mathrm{~S}_{1}\right)+$ $\mathrm{e}_{1}^{-}+\mathrm{e}_{2}^{-}$. These processes, whose contributions are calculated in detail for Fe and $\mathrm{Ca}$ by Bely-Dubau et al. (1982a) and Bely-Dubau et al. (1982b), are included in the calculation of synthetic spectra in the Yohkoh BCS analysis software. The atomic data files used in the BCS analysis software have excitation rate coefficients (integral over energy of excitation cross sections times maxwellian distributions) in the form

$\left\langle Z^{2} \Omega_{\mathrm{eff}}\right\rangle=\frac{C_{12}(Z-0.5)^{2} T_{\mathrm{e}}^{1 / 2} \exp \left(\Delta E / k_{\mathrm{B}} T_{\mathrm{e}}\right)}{8.63 \times 10^{-6}}$

where $C_{12}$ is the rate coefficient $\left(\mathrm{cm}^{3} \mathrm{~s}^{-1}\right)$ for excitation from the ground level (1) to the excited level (2), and $\Delta E$, the excitation energy, is given by a hydrogenic approximation, $\Delta E=$ $1.63 \times 10^{-11}(Z-0.5)^{2} \mathrm{erg}$. The more familiar temperatureaveraged collision strength $\Upsilon$,

$\Upsilon=\int \Omega(E) \exp \left(-E / k_{\mathrm{B}} T_{\mathrm{e}}\right) \mathrm{d} E$

( $\Omega=$ collision strength), is related by $\left\langle Z^{2} \Omega_{\text {eff }}\right\rangle=(Z-$ $0.5)^{2} \Upsilon$. The collisional excitation rate coefficients in the currently used FeXXV and CaXIX BCS atomic data files, which were originally compiled by one of us (JD), are from the distorted wave calculations of Bely-Dubau et al. (1982a) and Bely-Dubau et al. (1982b) (see a review of these data by Dubau 1984 and Kato \& Nakazaki 1989). The values of $\left\langle Z^{2} \Omega_{\text {eff }}\right\rangle$ are "effective", i.e. include a branching ratio to account for cascades within the $n=2$ levels as well as cascades from $n>2$ levels. The atomic data files also include rate coefficients for recombination (of importance for the $x-z$ lines) and inner-shell ionization (for line $z$ ).

Since these data files were compiled, there has been an increasing awareness of the importance of auto-ionizing resonances in the collisional excitation of He-like ions (see e.g. Mewe 1999). These resonances occur in atomic processes such as $\mathrm{He}\left(1 \mathrm{~s}^{2}\right)+\mathrm{e}_{1}^{-} \rightarrow \operatorname{Li}\left(1 \mathrm{~s} 2 \mathrm{ln}^{\prime} 1^{\prime}\right) \rightarrow \mathrm{He}(1 \mathrm{~s} 2 \mathrm{l})+\mathrm{e}_{2}^{-}$, i.e. a doubly excited state de-excites by auto-ionization to the upper level $1 \mathrm{~s} 21$ of one of the He-like ion lines. No resonance excitation occurs if the doubly excited Li-like ion is radiatively deexcited, i.e. dielectronic recombination occurs; this is increasingly likely for higher- $Z$ ions such as Fe and $\mathrm{Ca}$. Nevertheless, auto-ionizing resonances are important for $\mathrm{Fe}$ and $\mathrm{Ca}$, and calculations such as those by Faucher \& Dubau (1985) and Zhang \& Sampson (1987) have taken them into account.

In this paper, we used collision strengths and $\Upsilon$ values for Ca XIX and Fe XXV calculated by Kimura et al. (2000) with the relativistic $R$-matrix code, in which resonances are included by a close-coupling formalism. Further details are given by Rainnie (2002) and by Harra-Murnion et al. (1996) who used $R$-matrix atomic data for the case of SXV lines $w-z$. These data are already being used for the analysis of S XV spectra from flares and active regions by the Yohkoh BCS.

The collision rates of Kimura et al. (2000) do not allow for cascade contributions, so to include them it was necessary to use the existing data in the atomic data files for cascades. We also used existing data for radiative recombination

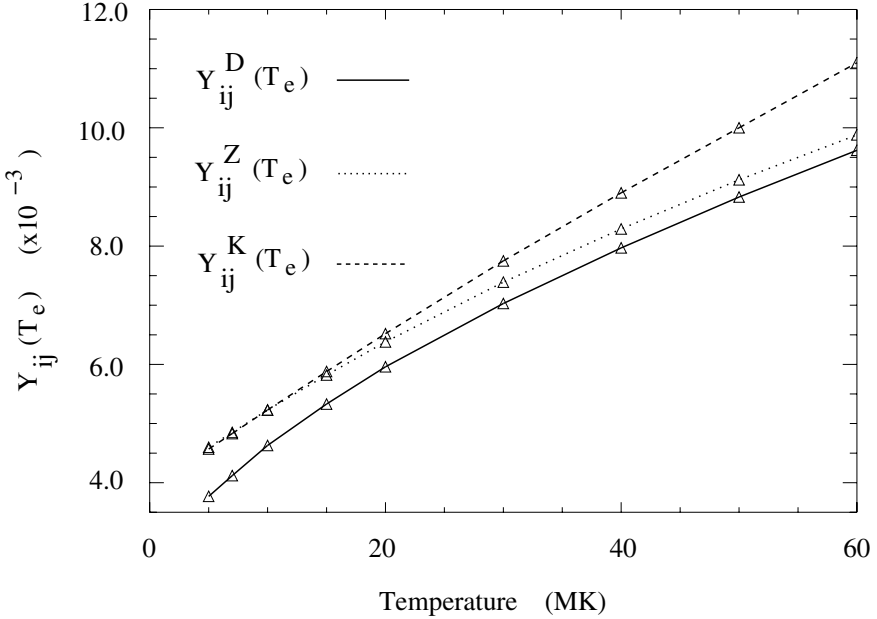

Fig. 1. Plots against temperature $T_{\mathrm{e}}$ of effective collisional rates $\Upsilon$ for excitation to the He-like Ca $1 \mathrm{~s} 2 \mathrm{p}^{1} \mathrm{P}_{1}$ level (upper level of CaXIX $w$ line). Data from Dubau $\left(\Upsilon_{i j}^{\mathrm{D}}\left(T_{\mathrm{e}}\right)\right.$, based on $Z^{2}\left\langle\Omega_{\mathrm{eff}}\right\rangle$ values: solid lines), Zhang \& Sampson (1987) $\left(\Upsilon_{i j}^{Z S}\left(T_{\mathrm{e}}\right)\right.$ : dotted lines) and Kimura et al. (2000) $\left(\Upsilon_{i j}^{\mathrm{K}}\left(T_{\mathrm{e}}\right)\right.$ : dashed lines) are compared.

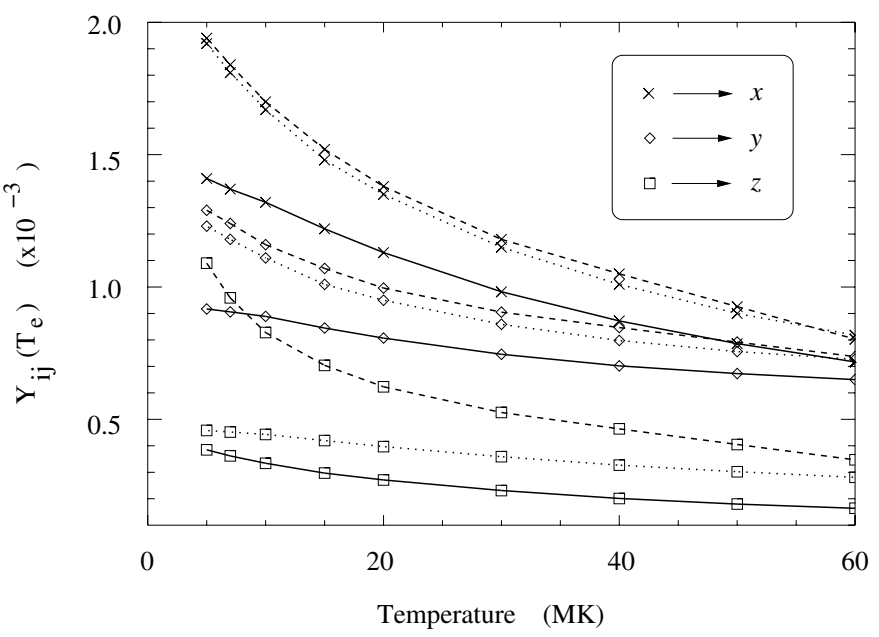

Fig. 2. Plots against $T_{\mathrm{e}}$ of effective collisional rates $\Upsilon$ from Dubau (solid lines), Zhang \& Sampson (1987) (dotted lines), and Kimura et al. (2000) (dashed lines) for excitation to the He-like $\mathrm{Ca} 1 \mathrm{~s} 2 \mathrm{p}^{3} \mathrm{P}_{2}$ (crosses: upper level of line $x$ ), $1 \mathrm{~s} 2 \mathrm{p}^{3} \mathrm{P}_{1}$ (diamonds: upper level of line $y$ ), and $1 \mathrm{~s} 2 \mathrm{~s}^{3} \mathrm{~S}_{1}$ levels (squares: upper level of line $z$ ).

for excitation of the $1 \mathrm{~s} 2 \mathrm{p}^{3} \mathrm{P}_{1,2}$ and $1 \mathrm{~s} 2 \mathrm{~s}{ }^{3} \mathrm{~S}_{1}$ levels and collisional ionization rates for excitation of the $1 \mathrm{~s} 2 \mathrm{~s}^{3} \mathrm{~S}_{1}$ level. Figures 1 and 2 show comparisons of $\Upsilon$ values plotted against $T_{\mathrm{e}}$ for excitation to the CaXIX levels $1 \mathrm{~s} 2 \mathrm{p}^{1} \mathrm{P}_{1}, 1 \mathrm{~s} 2 \mathrm{p}^{3} \mathrm{P}_{1}$, $1 \mathrm{~s} 2 \mathrm{p}^{3} \mathrm{P}_{2}$, and $1 \mathrm{~s} 2 \mathrm{~s}^{3} \mathrm{~S}_{1}$ (upper levels of lines $w, y, x$, and $z$ respectively). Kimura et al. (2000) also calculated $\Upsilon$ values for the $1 \mathrm{~s} 2 \mathrm{~s}^{1} \mathrm{~S}_{0}$ and $1 \mathrm{~s} 2 \mathrm{p}^{3} \mathrm{P}_{0}$ levels which are not illustrated here. The $\Upsilon_{s}$ shown in these figures which are used in existing Yohkoh BCS atomic data files ("Dubau") are those based on $\left\langle Z^{2} \Omega_{\text {eff }}\right\rangle$ values. For excitation to the CaXIX $1 \mathrm{~s} 2 \mathrm{p}^{1} \mathrm{P}_{1}$ (upper level of line $w$ ), $1 \mathrm{~s} 2 \mathrm{p}^{3} \mathrm{P}_{2}$ (line $x$ ), $1 \mathrm{~s} 2 \mathrm{p}^{3} \mathrm{P}_{1}$ (line $y$ ), and $1 \mathrm{~s} 2 \mathrm{~s}^{3} \mathrm{~S}_{1}$ (line $z$ ) at $T_{\mathrm{e}} \sim 13 \mathrm{MK}(1 \mathrm{MK}=$ $10^{6} \mathrm{~K}$ ), the Kimura et al. (2000) rates are respectively $12 \%$, $27 \%, 27 \%$, and a factor 2.4 higher than the Dubau values. 


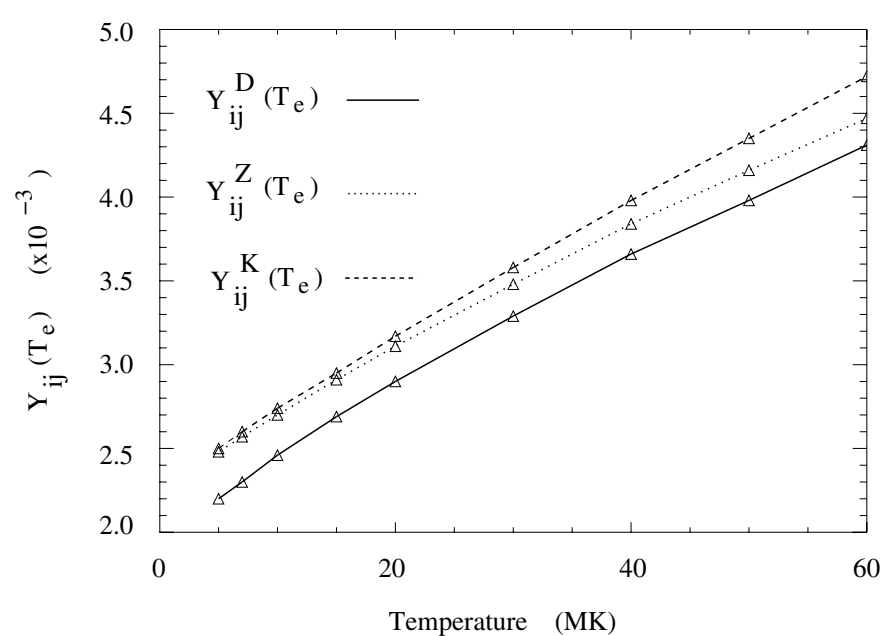

Fig. 3. Plots against $T_{\mathrm{e}}$ of effective collisional rates $\Upsilon$ from Dubau, Zhang \& Sampson (1987), and Kimura et al. (2000) for excitation to the He-like Fe $1 \mathrm{~s} 2 \mathrm{p}{ }^{1} \mathrm{P}_{1}$ level (upper level of Fe XXV $w$ line). See Fig. 1 for symbols.

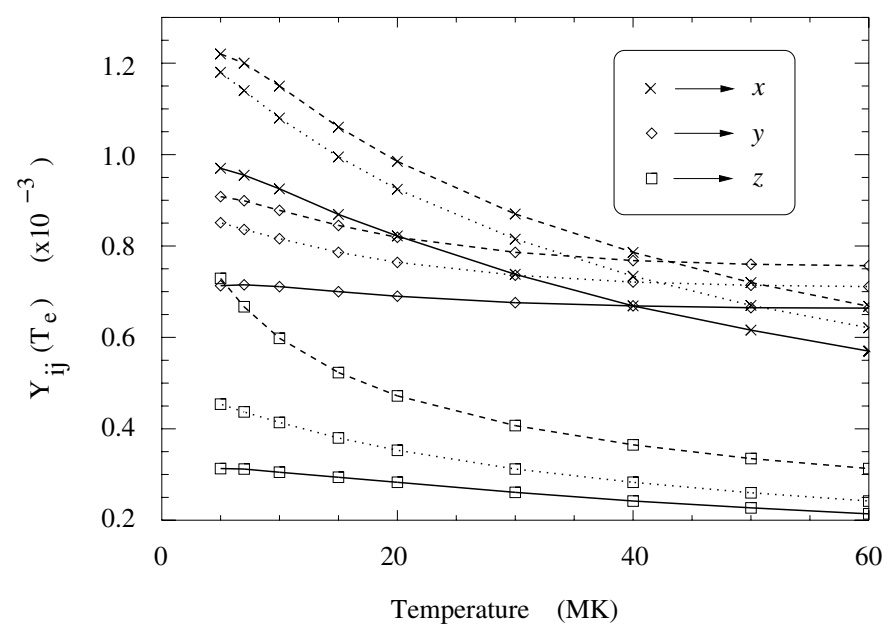

Fig. 4. Plots against $T_{\mathrm{e}}$ of effective collisional rates $\Upsilon$ from Dubau, Zhang \& Sampson (1987), and Kimura et al. (2000) for excitation to the He-like Fe $1 \mathrm{~s} 2 \mathrm{p}^{3} \mathrm{P}_{1}, 1 \mathrm{~s} 2 \mathrm{p}^{3} \mathrm{P}_{2}$, and $1 \mathrm{~s} 2 \mathrm{~s}{ }^{3} \mathrm{~S}_{1}$ levels (upper levels of Fe XXV $y, x$, and $z$ respectively). See Fig. 2 for symbols.

The Zhang \& Sampson (1987) rates are intermediate but very close to the Kimura et al. (2000) values for excitation to the $1 \mathrm{~s} 2 \mathrm{p}^{3} \mathrm{P}_{1}$ and $1 \mathrm{~s} 2 \mathrm{p}^{3} \mathrm{P}_{2}$ levels.

Figures 3 and 4 show similar plots for excitation to Fe XXV levels. In this case, for excitation to the Fe XXV $1 \mathrm{~s} 2 \mathrm{p}^{1} \mathrm{P}_{1}$ (upper level of line $w$ ), $1 \mathrm{~s} 2 \mathrm{p}^{3} \mathrm{P}_{2}$ (line $x$ ), $1 \mathrm{~s} 2 \mathrm{p}^{3} \mathrm{P}_{1}$ (line $y$ ), and $1 \mathrm{~s} 2 \mathrm{~s}{ }^{3} \mathrm{~S}_{1}$ (line $z$ ) at $T_{\mathrm{e}} \sim 25 \mathrm{MK}$, the Kimura et al. (2000) values are respectively higher than the Dubau values by $10 \%, 19 \%, 14 \%$, and $60 \%$, with the Zhang \& Sampson (1987) values intermediate.

Inclusion of either the Kimura et al. (2000) or the Zhang \& Sampson (1987) results shown in Figs. 1-4 will give rise to theoretical intensities of lines $w-z$ different from those derived using presently used atomic data. We synthesized $\mathrm{Ca}$ and Fe line spectra with Yohkoh BCS software using the excitation data illustrated in these figures as well as existing data for the dielectronic satellites which remain

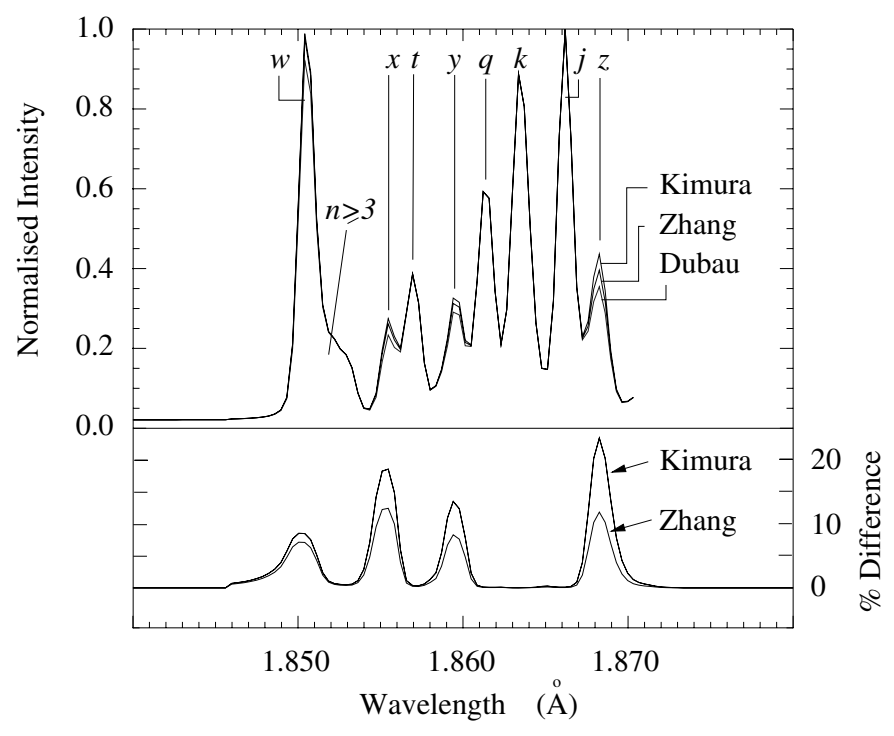

Fig. 5. Top panel: synthesized Fe XXV spectra (including Fe XXIV satellites) for $T_{\mathrm{e}}=15 \mathrm{MK}$ using effective collision strengths of Dubau (present BCS atomic data), Zhang \& Sampson (1987), and Kimura et al. (2000). Bottom panel: differences in flux, plotted against wavelength $(\AA)$, in the sense Kimura et al. (2000) minus Dubau (thick line) and Zhang \& Sampson (1987) minus Dubau (thin line). In order of increasing wavelength, the four lines in the bottom panel are $w, x, y$, and $z$. Lines $t$ and $q$ are inner-shell satellites, $j$ and $k$ and those marked $n \geq 3$ (which include $d 13$ and $d 15$ ) are dielectronic satellites.

unchanged. Figure 5 shows an Fe XXV spectrum with $T_{\mathrm{e}}=$ $15 \mathrm{MK}$ synthesized using the effective collision strengths of Dubau, Zhang \& Sampson (1987), and Kimura et al. (2000). The wavelength range (1.84-1.88 $\AA$ ) includes the Fe XXV lines $w-z$, most Fe XXIV satellites, and some Fe XXIII satellites. The differences between the spectra are very small. They are more clearly displayed in the bottom panel of Fig. 5; the maximum differences are between the Kimura et al. (2000) and Dubau calculations, amounting to $7.5 \%$ (line $w$ ), $17 \%$ (line $x$ ), $12 \%$ (line $y$ ), and $23 \%$ for line $z$. These differences are less than those between the $\Upsilon$ values for lines $x, y$, and $z$, owing to contributions made to the intensities of these lines by cascades and, to a smaller extent, recombination and (in the case of line $z$ ) inner-shell ionization. Even smaller differences occur for Ca XIX lines $w-z$.

The small differences made by using the Kimura et al. (2000) data in place of the original Dubau data will make small differences in values of $T_{\mathrm{e}}$ which are estimated by the satellite-to-resonance line ratios $j / w$ and $k / w$ in the case of Fe XXV spectra observed by the Yohkoh BCS or the SMM Bent Crystal Spectrometer. For Yohkoh Ca XIX spectra, $T_{\mathrm{e}}$ is determined by the ratio of the feature made up of the $d 13$ and $d 15$ satellites to line $w$. Lines $j$ and $k$ are included in the wavelength range of the $S M M$ spectrometer (though $j$ is blended with the Ca XIX $z$ line). With the Kimura et al. (2000) and Zhang \& Sampson (1987) data, the satellite-to- $w$ line ratio is decreased, so that for a given observed ratio, the value of $T_{\mathrm{e}}$ is decreased. We illustrate the differences between $T_{\mathrm{e}}$ estimates using the present (Dubau), Zhang \& Sampson (1987), 


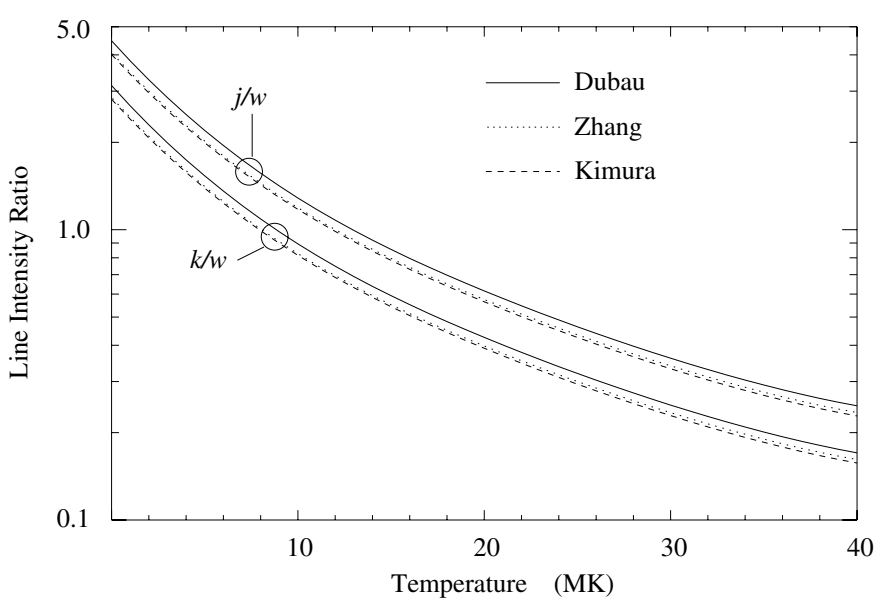

Fig. 6. Line ratios of FeXXIV satellites $j$ and $k$ to FeXXV line $w$ using effective rate coefficients from Dubau (present BCS atomic data), Zhang \& Sampson (1987), and Kimura et al. (2000).

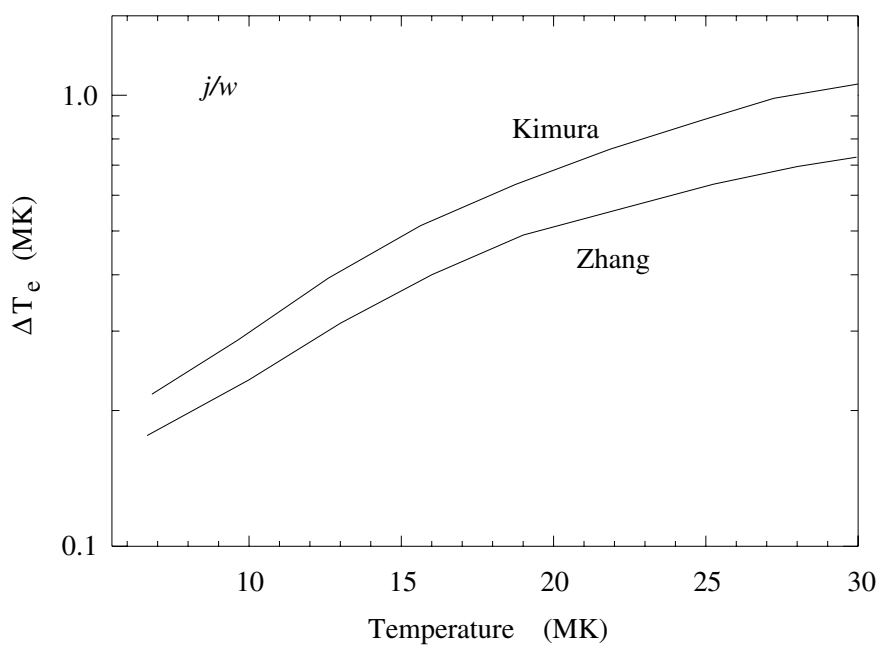

Fig. 7. Decreases in temperature $\left(\Delta T_{\mathrm{e}}\right)$ measured from a given observed Fe XXV $j / w$ ratio, through using effective collision strengths from Zhang \& Sampson (1987) and Kimura et al. (2000) compared with Dubau (present atomic data).

and Kimura et al. (2000) atomic data for the case of Fe XXV spectra - the differences are approximately the same for Ca XIX spectra. The $j / w$ ratios are plotted against $T_{\mathrm{e}}$ in Fig. 6 and the consequent decreases in $T_{\mathrm{e}}\left(\Delta T_{\mathrm{e}}\right)$ are plotted in Fig. 7. As can be seen, $\Delta T_{\mathrm{e}}$ is between 0.5 and $1 \mathrm{MK}$ for likely solar flare temperatures.

An important and still unresolved issue in He-like Fe spectra is the fact that the intensities of lines $y$ and $z$ in solar flare spectra are more intense than predicted by the calculations used in the BCS software (e.g. Lemen et al. 1984; Doschek \& Tanaka 1987). The differences for Fe XXV spectra amount to about $50 \%$ for $y$ and $20 \%$ for $z$. The inner-shell satellites $q$ and $r$ in flare Ca XIX and Fe XXV spectra are also overintense. As indicated earlier, the Kimura et al. (2000) atomic data (and to a smaller extent the Zhang \& Sampson (1987) data) predict greater intensities of lines $y$ and $z$ through the larger values of $\Upsilon$ for excitation to the upper levels of these lines.

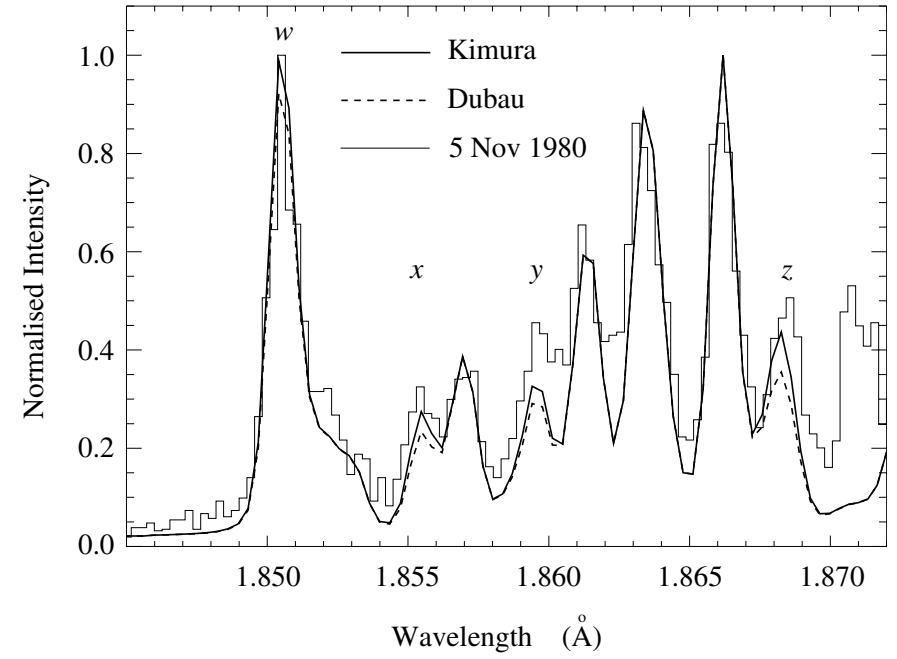

Fig. 8. SMM BCS Fe XXV spectrum from near the peak of the flare on 1980 November 5 (histogram) compared with synthetic spectra using excitation rates from Dubau (dashed line) and Kimura et al. (2000) (solid line). The discrepancy between observed and calculated spectra is reduced by a factor $\sim 2$ for line $z$ using the Kimura et al. (2000) data but by very little for line $y$.

A comparison was therefore made between synthetic spectra from the Dubau and Kimura et al. (2000) atomic data and solar flare CaXIX and Fe XXV spectra from the SMM Bent Crystal Spectrometer. Figure 8 shows the observed SMM spectrum near the peak of the large flare on 1980 November 5 together with calculated spectra for $T_{\mathrm{e}}=15 \mathrm{MK}$, estimated from the $j / w$ and $k / w$ ratios. The discrepancy between the observed and calculated intensities of the $z$ line is reduced by about a factor 2, though only by a small amount for the $y$ line. Figure 9 shows observed and synthetic Ca XIX. In this case, there is reasonably good agreement for line $z$ but the observed intensity of line $y$ remains greater than predicted for both the Dubau and Kimura et al. (2000) atomic data. There is however a possibility that this discrepancy has an instrumental origin connected with the crystal reflectivity at the position of the $y$ line. This seems to be borne out by Yohkoh BCS spectra which for flares located in the Sun's southern hemisphere show the $x$ and $y$ lines clearly; the discrepancy for line $y$ does not seem to be in evidence for these flares.

Thus, although the Kimura et al. (2000) excitation rates slightly improve the agreement between theoretical and observed solar flare intensities of FeXXV lines $y$ and $z$, the discrepancies persist. One can only speculate on the reasons for this. One might be that unaccounted-for satellites or even extraneous lines of another element are responsible for these discrepancies, possibly like the Be-like ion satellites which contribute to satellite $k$ in the case of He-like Ar spectra (Bitter et al. 2002). Another less likely reason is the remote possibility of neutral $\mathrm{H}$ atoms somehow diffusing into the hot flare plasma and persisting long enough to give rise to change exchange processes which favour excitation of the triplet lines, as found in tokamak spectra (Peacock et al. 1996). 


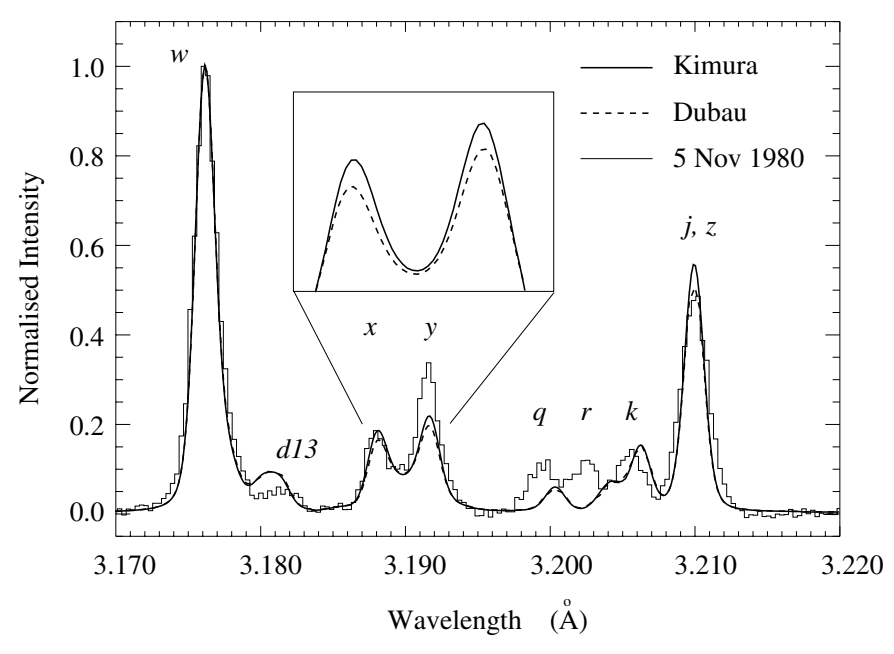

Fig. 9. SMM BCS Ca XIX spectrum from near the peak of the flare on 1980 November 5 (histogram) compared with synthetic spectra using excitation rates from Dubau and Kimura et al. (2000). The differences for the CaXIX $x$ and $y$ lines are shown in detail in the inset. The discrepancy in the $y$ line may be instrumental in origin (see text).

\section{EBIT Fe XXV spectra}

Another means of distinguishing between the sets of excitation data considered here is offered by Fe XXV spectra obtained from the Lawrence Livermore National Laboratory's electron beam ion trap (EBIT) (Brown et al. 1989). The measurements were made by trapping Fe ions in an electrostatic well and ionizing them to the helium-like stage $\left(\mathrm{Fe}^{+24}\right)$ by an electron beam with precisely determined energy. The resulting Fe XXV spectra were observed by a curved crystal spectrometer. In one of the experiments done by Brown et al. (1989), spectra were obtained with an electron beam energy of $6.86 \pm 0.01 \mathrm{keV}$, slightly above the excitation energy of the Fe XXV $w$ line. This energy was chosen so as not to coincide with an auto-ionizing resonance and is also lower than any $n=3$ level so that cascade transitions are not possible. In addition, dielectronic satellites are not present in these spectra as their excitation energies are lower $\left(E_{\mathrm{s}} \sim 4.6 \mathrm{keV}\right.$ for $n=2$ satellites). Also, the ion trap contained very few Li-like Fe ions, so that the inner-shell satellites $q, r, s$, and $t$ are very weak. However, as the spectrometer viewed the trap at $90^{\circ}$ to the electron beam, there are polarization effects for lines $w, x$, and $y$, though not line $z$. When corrected for polarization effects, the ratios of the $x, y$, and $z$ lines to line $w$ (Table 1) are smaller than solar flare or tokamak values (Fig. 8), reflecting the absence of cascade excitation and recombination of these lines.

The EBIT intensity ratios may be compared with line intensities derived from Kimura et al. (2000)'s collision strengths at an energy of $6.86 \mathrm{keV}$, close to the measured beam energy, and with those of Bely-Dubau et al. (1982a) and Zhang \& Sampson (1987) (Table 1). In calculating the theoretical ratios from Kimura et al. (2000)'s data, we allowed for the fact that de-excitation of the $1 \mathrm{~s} 2 \mathrm{p}^{3} \mathrm{P}_{2}$ level is both to the ground level $(82 \%)$ and to the $1 \mathrm{~s} 2 \mathrm{~s}^{3} \mathrm{~S}_{1}$ level $(18 \%)$ and that radiative de-excitation of the $1 \mathrm{~s} 2 \mathrm{p}^{3} \mathrm{P}_{0}$ level occurs to the $1 \mathrm{~s} 2 \mathrm{~s}^{3} \mathrm{~S}_{1}$ level. From the table it can be seen that the calculated ratios all agree with the measured ratios to within uncertainties, so we are unable to state which calculation is in better agreement with the measurements. Note that the discrepancies noted for the $y$ and $z$ lines in solar flare spectra (Sect. 3) do not seem to occur for these EBIT spectra.

\section{S XV spectra}

The Yohkoh BCS atomic database already includes $R$-matrix rate coefficients for $\mathrm{SXV} w-z$ lines (Harra-Murnion et al. 1996). Provision for sensitivity of lines $x-z$ to electron density $N_{\mathrm{e}}$ (which occurs at $N_{\mathrm{e}} \gtrsim 10^{13} \mathrm{~cm}^{-3}$ through the excitation of the metastable $1 \mathrm{~s} 2 \mathrm{~s}^{3} \mathrm{~S}_{1}$ level to the $1 \mathrm{~s} 2 \mathrm{p}^{3} \mathrm{P}$ levels) is made in the software to allow for the possibility of high densities in some flares. These large densities occur in tokamak plasmas such as those produced at the UK Atomic Energy Authority's COMPASS-D device at Culham Laboratory. By suitable adjustment of instrumental parameters it is possible to synthesize S XV spectra for comparison with observed spectra. COMPASS-D spectra obtained without beam heating (Peacock et al. 1996) observed with a high-resolution spectrometer are particularly suitable as the ion temperatures are relatively low $\left(T_{\text {ion }} / T_{\mathrm{e}} \sim 0.4\right)$. This allows spectral features to be much better resolved than in solar-flare spectra, including S XIV satellite $k$, some $n=4$ satellites and the $d 13 / d 15$ feature which in Yohkoh BCS channel 4 flare spectra are at least partly blended with line $z$ or line $w$. Furthermore, as $T_{\mathrm{e}}$ and $N_{\mathrm{e}}$ can be independently measured, a comparison of COMPASS-D and synthetic spectra provides a "benchmark" test of the analysis software producing the synthetic spectra.

Figure 10 shows a COMPASS-D S XV spectrum compared with a BCS synthetic spectrum with $T_{\mathrm{e}}=8.1 \mathrm{MK}$ and $N_{\mathrm{e}}$ set equal to $8 \times 10^{13} \mathrm{~cm}^{-3}$, as determined by laser-scattering measurement. The instrumental line profile width in the synthetic spectrum is set equal to that of the spectrometer used. There is very close agreement between the two spectra, the main differences being in the wavelengths of the $x, y$ and $z$ lines and the $n=4$ satellite feature (residuals are indicated in the plot). The observed and fitted width of line $w$ agree particularly well. This is a validation of the wavelengths of the many high$n$ satellites (transitions $1 \mathrm{~s}^{2} n l-1 \mathrm{~s} 2 \mathrm{p} n l, n$ up to 6 ) included in the atomic database which occur on the long-wavelength side of $w$, converging on $w$ for increasing $n$. Although these satellites are individually weak, their cumulative effect is important for the total intensity and width of the blended $w$ line feature (see Bely-Dubau et al. 1979). A correct width for this feature is of importance for investigations using Yohkoh BCS spectra of nonthermal velocities such as are observed in flares.

A typical SXV spectrum during a flare observed by the Yohkoh BCS is shown in Fig. 11, together with best-fit synthetic spectrum obtained in the low-density limit using the Yohkoh BCS software with presently used atomic database to illustrate how this satisfactorily describes flare spectra.

\section{Fe XXVI spectra}

Very large X-ray flares may produce significant Fe XXVI line emission, which was observed by both the Yohkoh and SMM 
Table 1. Calculated and EBIT (beam energy $6.9 \mathrm{keV}$ ) Fe XXV ratios.

\begin{tabular}{ccccc}
\hline \hline Ratio & This paper & Bely-Dubau et al. $^{a}$ & Zhang \& Sampson $^{b}$ & EBIT \\
\hline$I(x) / I(w)$ & 0.198 & 0.19 & 0.210 & $0.18 \pm 0.03$ \\
$I(y) / I(w)$ & 0.215 & 0.20 & 0.211 & $0.25 \pm 0.04$ \\
$I(z) / I(w)$ & 0.248 & 0.23 & 0.237 & $0.24 \pm 0.06$ \\
\hline${ }^{a}$ Bely
\end{tabular}

${ }^{a}$ Bely-Dubau et al. (1982a).

${ }^{b}$ Zhang \& Sampson (1987).

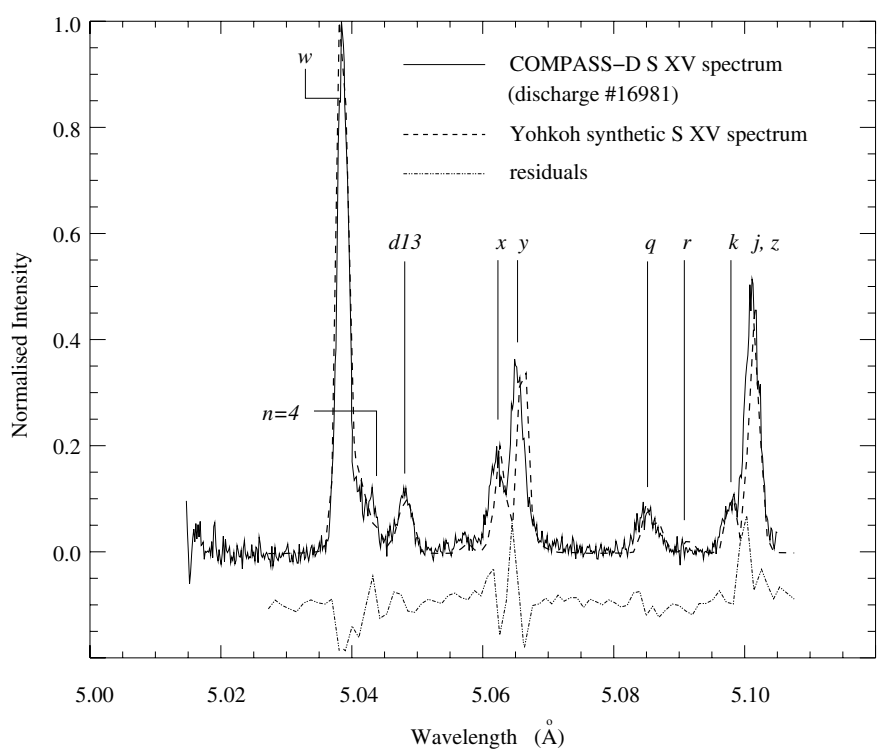

Fig. 10. S XV spectrum from COMPASS-D tokamak discharge No. \#16981 compared with a synthetic spectrum from the Yohkoh software (smooth curve) and residuals shown as negative values. The synthetic spectrum was calculated with Yohkoh BCS software using laboratorydetermined values of electron temperature $\left(T_{\mathrm{e}}=8.1 \mathrm{MK}\right)$ and density $\left(8 \times 10^{13} \mathrm{~cm}^{-3}\right)$. Principal S XV lines and S XIV satellites (notation of Gabriel 1972) are indicated.

crystal spectrometers. In the case of the Yohkoh BCS, over 70 flares were identified in a study by Pike et al. (1996) having identifiable (but in all cases very weak) Fe XXVI Ly $\alpha$ line emission. The probability of such emission is clearly related to the temperature derived from Fe XXV spectra and GOES flare class. Pike et al. (1996) analyzed Fe XXVI line emission, deriving $T_{\mathrm{e}}$ from the ratio of the Fe XXV satellite feature known as $J$ (Dubau et al. 1981) to the Fe XXVI Ly $\alpha_{1}$ line over a single time period ranging from 1 to $17 \mathrm{~min}$ long, including the time of maximum Fe XXV emission in each flare. For the more intense of these flares, it should be possible to derive $T_{\mathrm{e}}$ for several times, as is done for Fe XXV lines. An error in one of the analysis routines has prevented this until now. The error (pointed out by N. Nitta) is due to the fact that the intensities of the Fe XXV satellites including the $J$ feature were calculated in synthetic spectra by taking the intensities to be proportional to the number density of $\mathrm{Fe}^{+24}$, not $\mathrm{Fe}^{+25}$, ions. As with Fe XXIV dielectronically formed satellites near the Fe XXV $w$ line, the Fe XXV satellite-to-Fe XXVI Ly $\alpha$ line ratio is independent of ionization stages.

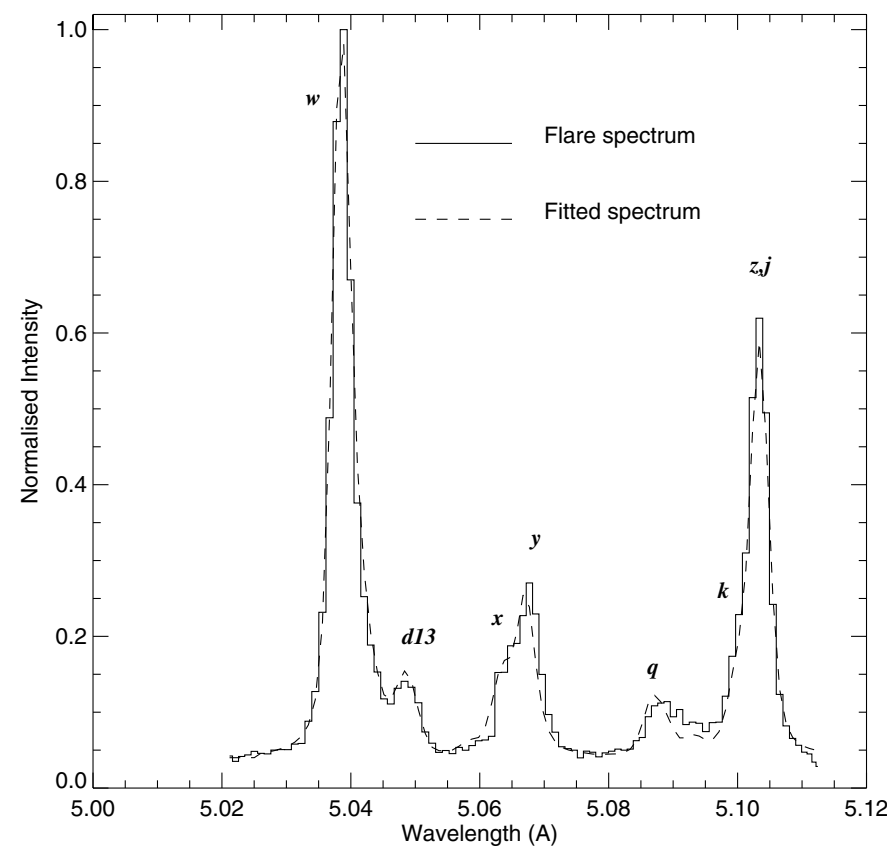

Fig. 11. S XV spectrum from the decay stage of a large disk flare on 1992 February 27 at 13:43 UT (integration time 30 s) observed by the Yohkoh BCS, together with best-fit synthetic spectrum in the low-density limit using the BCS software and database. Principal $\mathrm{S}$ XV lines and S XIV satellites are indicated.

A correction was made for this by changing data in the $\mathrm{Fe}$ ion balance file read by BCS software ${ }^{1}$. With the modified ion balance files it is now possible to analyze flares having Fe XXVI line emission routinely to get $T_{\mathrm{e}}$ and other parameters. As an illustration, Fig. 12 shows synthetic Fe XXVI spectra for a range of temperatures, and comparison with synthetic spectra given by Dubau et al. (1981) shows how the software now correctly predicts the variation of the Fe XXV satellite feature $J$ relative to the Fe XXVI Ly $\alpha$ lines. Nearly all of the observed BCS Fe XXVI spectra are very weak (Pike et al. 1996); Fig. 13 shows one of the more intense examples together with best-fit synthetic spectrum obtained with modified Fe ion balance file.

\section{Summary}

The effects of using improved atomic data for the Fe XXV and Ca XIX X-ray spectra have been examined by synthesizing spectra and comparing with $S M M$ data. The data, calculated

\footnotetext{
1 The web site www.gsfc.nasa.gov/rhessi/ phillips/BCS/ gives details about this file and procedure needed to change it. Data for the Fe XXV and Ca XIX spectra described in Sect. 3 are also given on this web site.
} 


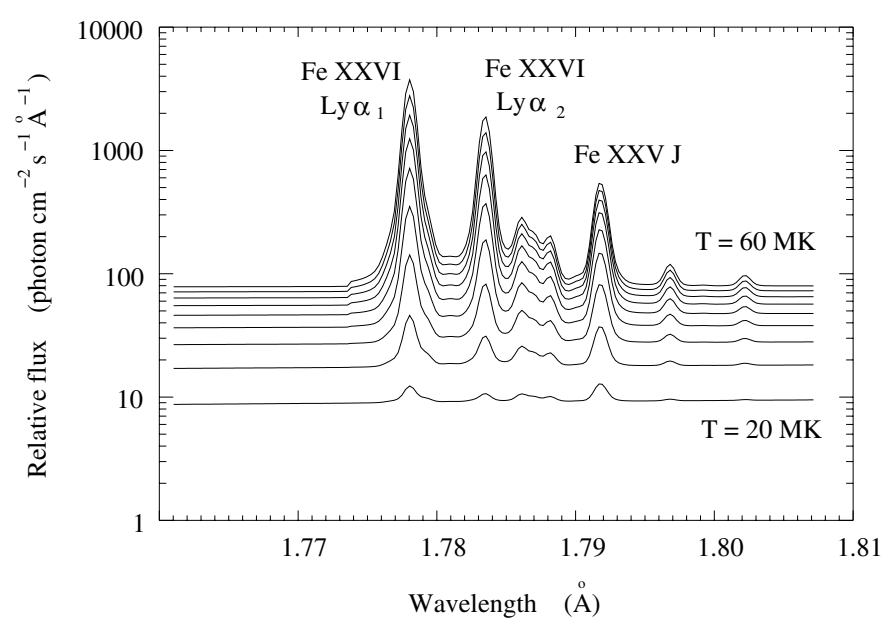

Fig. 12. Fe XXVI spectra synthesized using Yohkoh BCS software for $T_{\mathrm{e}}=20 \mathrm{MK}$ to $60 \mathrm{MK}$ in $5 \mathrm{MK}$ steps. The flux scale is relative, in photons (not erg) units. For smaller temperatures, the Fe XXV satellite feature $J$ is relatively more intense.

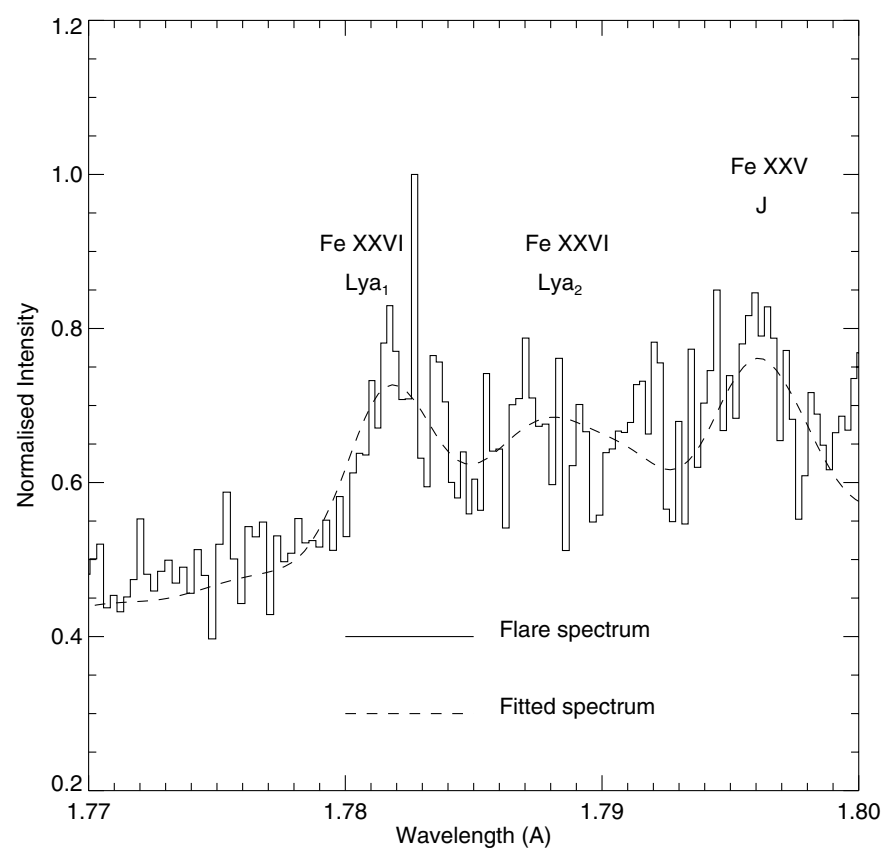

Fig. 13. Fe XXVI spectrum observed by the Yohkoh BCS from the peak of the large flare on 1991 December 16 at 04:57 UT (integrated over 30 s) with best-fit spectrum using the BCS software and database, with data file modified as described in the text. Principal lines are indicated.

by Kimura et al. (2000), are in the form of $R$-matrix collision strengths and temperature-averaged collision strengths with $T_{\mathrm{e}}$ for transitions from the He-like $\mathrm{Fe}$ and $\mathrm{Ca}$ ground states to $n=2$ levels. The presently used data for cascade transitions and excitation rates by recombination and inner-shell ionization are used with the Kimura et al. (2000) data. The effects of the inclusion of the new data are found to be very small. The well-known discrepancy between the observed and predicted intensities of the Fe XXV lines $y$ and $z$ are slightly reduced in spectra synthesized with the Kimura et al. (2000) collisional rate data. Use of the new data leads to a slight increase in the $w$ line intensity and so to a reduction, of up to
$1 \mathrm{MK}$, in the measured $T_{\mathrm{e}}$ for a given observed ratio of satelliteto-line $w$. This might be of significance for certain purposes, e.g. calculating Spitzer heat conduction times which depend on a high power of $T_{\mathrm{e}}$. The He-like ion line intensities calculated with the present and new collisional rates both agree with EBIT Fe XXV intensities to within experimental uncertainty, so it is not possible to state whether the new data are an improvement in this case. The Yohkoh BCS software was applied to a high-resolution tokamak S XV spectrum with low ion temperature (giving narrow line profiles). The agreement is good, so validating the wavelengths of high- $n$ satellites included in the atomic data for this ion at least. Finally, a long-standing error in the software for the calculation of Fe XXVI line spectra is corrected, and a method of using the data files to analyze BCS flare data where this line emission is present is illustrated.

The Yohkoh database may be of use for high-resolution spectrometers which are being planned for forthcoming nonsolar X-ray missions such as Constellation- $X$ and Astro-E. These instruments will be capable of resolving the Fe XXIV satellite structure and so be able to diagnose the emitting plasmas in the same way as solar flare plasmas have been analyzed with the spectrometers on Yohkoh and SMM and similar instruments. Eventually, the data could be incorporated into atomic data analysis packages such as Chianti and MEKAL for routine analysis of any X-ray-emitting plasma.

Acknowledgements. This research was performed while KJHP held a National Research Council Research Associateship award at NASA Goddard Space Flight Center, and was in part fulfillment of the Ph.D. examination requirements of JAR at The Queen's University, Belfast. LKH is grateful to the UK Particle Physics and Astronomy Research Council for the award of an Advanced Fellowship, and FPK is grateful to AWE Aldermaston for the award of a William Penney Fellowship. We thank I. M. Melnick (Culham Science Centre, UK) who produced the S XV spectrum shown in Fig. 10, and N. Nitta for originally pointing out the error in Fe XXVI line analysis. We also thank J. R. Lemen and D. M. Zarro who originally designed the user-friendly Yohkoh BCS software package which has been extensively used over the past ten years. C. Brown (NRL) kindly supplied the EBIT spectra for us; we are grateful for his advice on them.

\section{References}

Acton, L. W., Finch, M. L., Gilbreth, C. W., et al. 1980, Sol. Phys., 65, 53

Arnaud, M., \& Raymond, J. 1992, ApJ, 398, 394

Bely-Dubau, F., Dubau, J., Faucher, P., \& Gabriel, A. H. 1982a, MNRAS, 198, 239

Bely-Dubau, F., Faucher, P., Steenman-Clark, L., et al. 1982b, MNRAS, 201, 1155

Bely-Dubau, F., Gabriel, A. H., \& Volonté, S. 1979, MNRAS, 189, 405

Bitter, M., Hill, K. W., Roquemore, A. L., et al. 2002, Princeton Plasma Physics Laboratory Preprint

Brown, C. M., Feldman, U., Daschek, G. A., et al. 1989, Phys. Rev. A, 40, 4089

Culhane, J. L., Bentley, R. D., Hiei, E., et al. 1991, Sol. Phys., 136, 89

Doschek, G. A., \& Tanaka, K. 1987, ApJ, 323, 799

Dubau, J., Gabriel, A. H., Loulergue, M., Steenman-Clark, L., \& Volonté, S. 1981, MNRAS, 195, 705 
Dubau, J. 1984, Eighth International Colloquium Ultraviolet and X-ray Spectroscopy of Astrophysical and Laboratory Plasmas, Proc. IAU Colloq., 86, 36

Faucher, P., \& Dubau, J. 1985, Phys. Rev. A, 31, 3672

Gabriel, A. H. 1972, MNRAS, 160, 99

Harra-Murnion, L. K., Phillips, K. J. H., Lemen, J. R., et al. 1996, A\&A, 308, 670

Inal, M. K., \& Dubau, J. 1987, J. Phys. B, 20, 4221

Kato, T., \& Nakazaki, S. 1989, Atom. Data Nucl. Data Tabl., 42, 313

Kepa, A., Sylwester, J., \& Bentley, R. D. 1999, Nineth European Meeting on Solar Physics, Magnetic Fields and Solar Processes, Florence (ESA SP-448), 835

Kimura, E., Nakazaki, S., Berrington, K. A., \& Norrington, P. H. 2000, J. Phys. B., 33, 3449
Lemen, J. R., Phillips, K. J. H., Cowan, R. D., Hata, J., \& Grant, I. P. 1984, A\&A, 135, 313

Mariska, J. T., \& Doschek, G. A. 1997, ApJ, 485, 904

Martens, P. C. H., \& Cauffman, D. P. (ed.) 2002, Multi-Wavelength Observations of Coronal Structure and Dynamics, Yohkoh Tenth Anniversary Meeting (Pergamon)

Mewe, R. 1999, in X-ray Spectroscopy in Astrophysics, EADN School X, Amsterdam, ed. J. van Paradijs, \& J. A. M. Bleeker (Berlin: Springer), Lect. Notes Phys., 520, 109

Peacock, N. J. 1996, Ap\&SS, 237, 341

Pike, C. D., Phillips, K. J. H, Lang, J., et al. 1996, ApJ, 464, 487

Rainnie, J. A. 2002, Ph.D. Thesis, The Queen's University Belfast

Zhang, H., \& Sampson, D. H. 1987, ApJS, 63, 487 